\title{
Dancing Bunches at the Tevatron
}

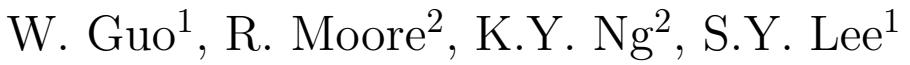 \\ ${ }^{1}$ Department of Physics, Indiana University, Bloomington, IN 47405 \\ ${ }^{2}$ Fermi National Accelerator Laboratory, P.O. Box 500, Batavia, IL 60510
}

(February, 2003)

\begin{abstract}
A train of uncoalesced proton bunches in consecutive $\mathrm{rf}$ buckets was seen executing synchrotron oscillations in the Tevatron at $150 \mathrm{GeV}$ over 15 minutes without appreciable filamentation. Analysis of the collected data shows two peaks in the fast Fourier transform (FFT), corresponding to two oscillatory motions. The lower-frequency motion is uncorrelated among the bunches and resembles the motion in an oscillatory potential. It is identified with the synchrotron motion. The higher-frequency motion is correlated among the bunches and resembles motion driven by an oscillating source.
\end{abstract}




\section{The Phenomenon}

Longitudinal bunch oscillations were observed in the Tevatron. The arrival times of the bunches were found moving around some center points for hours without deteriorating the beam. Hence, this phenomenon has been called "dancing bunches".

Here, we analyze two sets of experimental beam-profile data collected recently from a resistive-wall monitor and a 2 giga-sample-per-second digital mountain-range-display oscilloscope. The machine parameters for these experiments are listed in Table I. In the first experiment on September 21, 2002, 28 bunches in successive rf buckets were recorded in 1024 traces for a duration of about 1000 seconds. The horizontal scale of the bunch profile was $1 \mathrm{~ns}$ per data point and the separation between successive traces was 100 turns. The acquisition started from 1 to $1011 \mathrm{~s}$ after injection. The second experiment was performed on January 12, 2003. This time each trace contained 14 bunches in successive rf buckets. The data points were 0.5 ns apart in 1024 traces with 40-turn separation.

Table I: Tevatron Parameters.

\begin{tabular}{|lcl|}
\hline \hline Parameters & Notations & Values \\
\hline Average radius of Tevatron & $\mathrm{R}$ & $1000 \mathrm{~m}$ \\
Horizontal tune & $\nu_{x}$ & 20.583 \\
Vertical tune & $\nu_{z}$ & 20.575 \\
Chromaticities & $\xi_{x}, \xi_{z}$ & $8.0,8.0$ \\
Momentum compaction factor & $\alpha_{c}$ & 0.00283 \\
Revolution frequency & $f_{0}$ & $47713 \mathrm{~Hz}$ \\
Rf harmonic & $h_{r f}$ & 1113 \\
RF voltage & $V_{0}$ & $\sim 1.1 \mathrm{MV}$ \\
Energy & $E_{0}$ & $150 \mathrm{GeV}$ \\
Longitudinal tune & $\nu_{s}$ & $\sim 0.00192(\sim 91.5 \mathrm{~Hz})$ \\
\hline \hline
\end{tabular}

Figure 1 shows the longitudinal oscillatory motions of the 14 bunches in the second experiment for the first $0.10 \mathrm{sec}$. We see that not every bunch dances in the same way; it appears that they have different oscillating amplitudes and different phases. These were uncoalesced bunches injected from the Main Injector, each populated with typically $1 \times 10^{10}$ protons. This train of bunches is usually used for machine tune-up and is not designed for colliding beam physics.

Most of the analysis results are similar for the two experiments. Comparison is made when the results differ. 


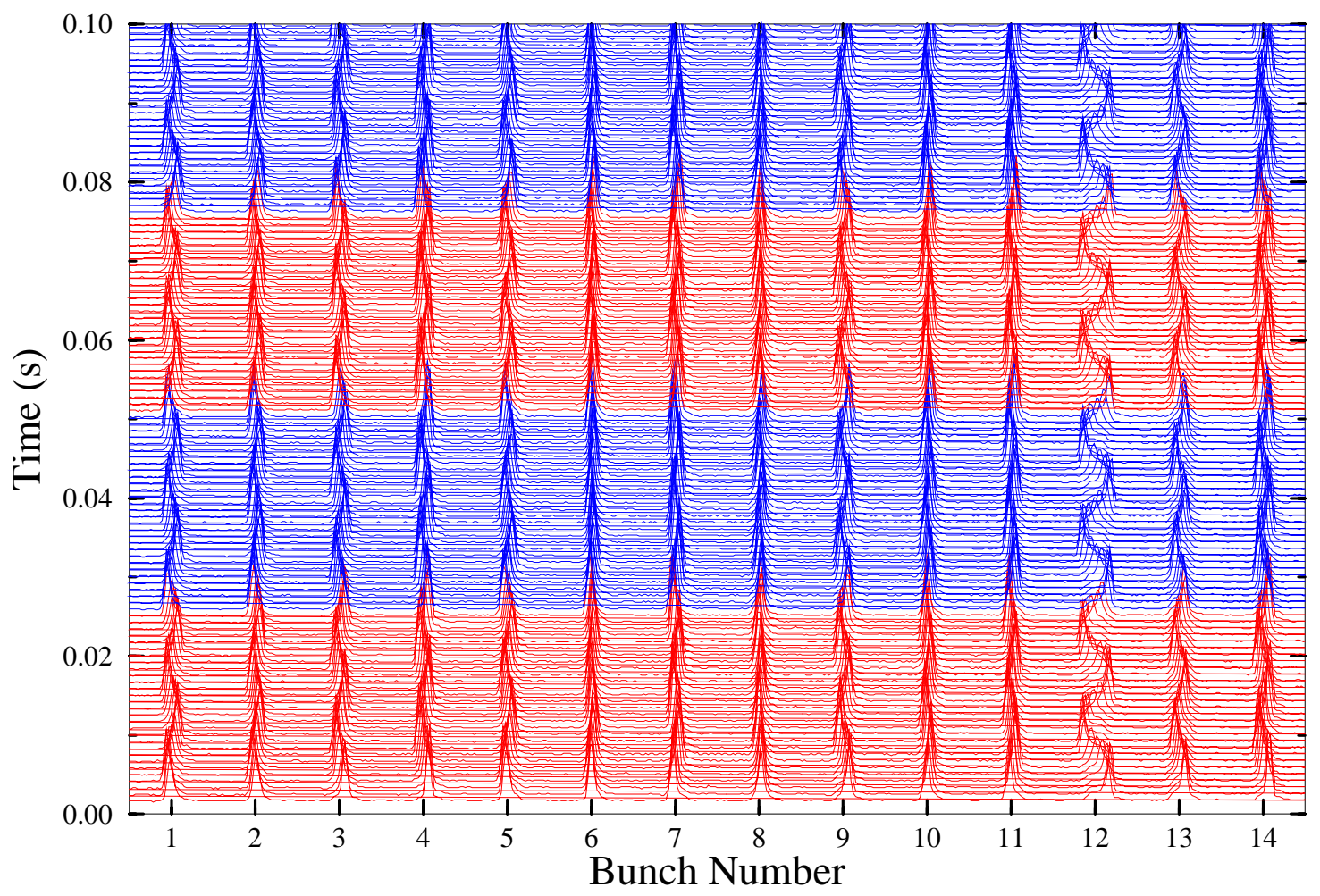

Figure 1: (color) Longitudinal profile of the centers-of-mass of the 14 uncoalesced bunches in the second experiment, collected $184 \mathrm{~s}$ after injection into the Tevatron. What is shown is the first $0.10 \mathrm{~s}$ of the recording. There are about 119 traces and they are 40 successive revolution turns apart.

\section{Particle Distribution and the Oscillation Frequencies}

Figure 2 shows a typical bunch profile in the first experiment fitted to a Gaussian distribution. Standard statistical analysis was applied. For each bunch in the first experiment, we first found the peak point, and used eight points around the peak (12 points in the second experiment) to calculate the bunch center $\langle t\rangle$ and rms bunch length $\sigma_{t}$. Too many points included in the analysis will lead to unrealistic large $\sigma_{t}$. On the other hand, too few points included will lead to large error bars. Fast Fourier Transform (FFT) was next performed on the 1,024 $\langle t\rangle$ values for each bunch in one file. Figure 3 shows a typical FFT result. One sees from Fig. 3 that there are two oscillation frequencies of $\langle t\rangle$. The first one is sharp and corresponds to the longitudinal synchrotron frequency of $\sim 88 \mathrm{~Hz}$. The second one is relatively broader, less intense, and peaks about $2 \sim 4$ times the synchrotron frequency. The FFT spectra of all 14 bunches in the 11 data files of the second experiment are shown in Fig. 4. The 11 data files* correspond to data taken $0,93,184,275,369,459,549,639,730,821$ and 911 s after injection into the Tevatron from the Main Injector at $150 \mathrm{GeV}$. We see that there is a strong variation

${ }^{*}$ These files are named data_xxx.txt where xxx represents the number of seconds recorded after injection. 


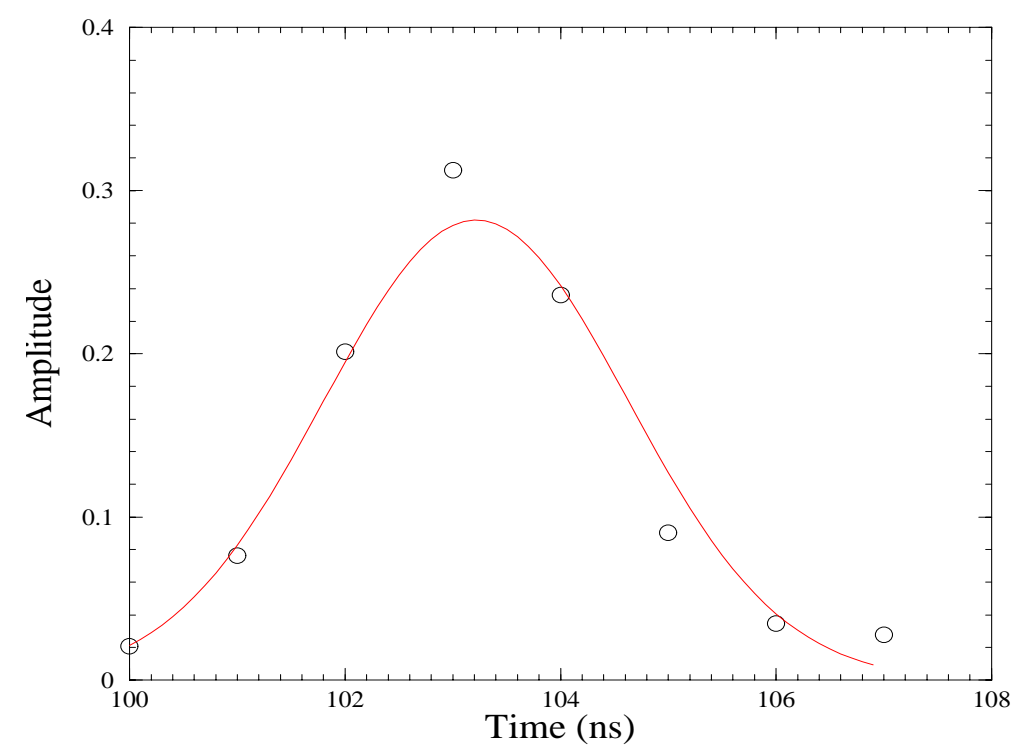

Figure 2: (color) Profile of the first bunch in the first column of file data_000.dat of the first experiment. The normalized experimental data points around the peak are represented by circles, from which the mean position $\langle t\rangle$ and rms length $\sigma_{t}$ of the bunch are computed. The red curve shows the Gaussian distribution corresponding to the computed $\sigma_{t}$ and $\langle t\rangle$.

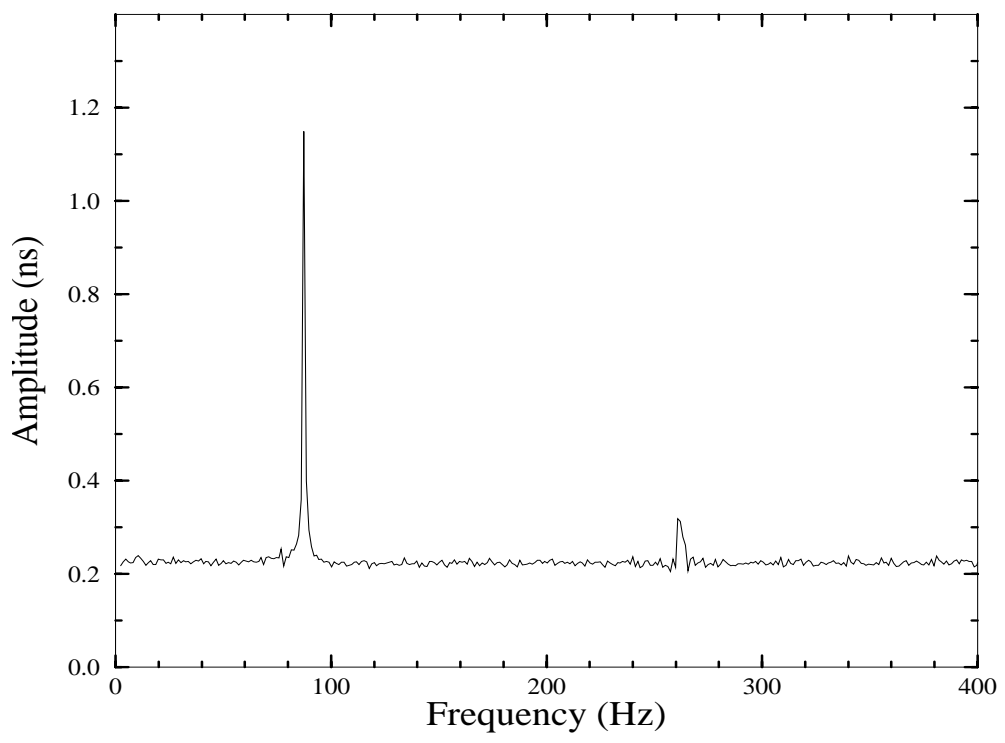

Figure 3: A typical FFT result of the centroid of a bunch in the second experiment. Besides a very sharp peak at $\sim 88 \mathrm{~Hz}$ for the synchrotron oscillation, there is another broader peak at $\sim 260 \mathrm{~Hz}$, which is not an harmonic of the synchrotron oscillation. 
FFT Spectrum of Bunch 1

FFT Spectrum of Bunch 2

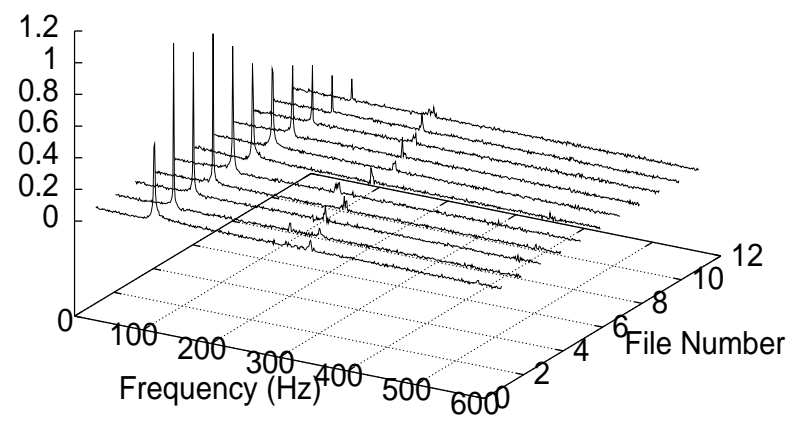

FFT Spectrum of Bunch 3

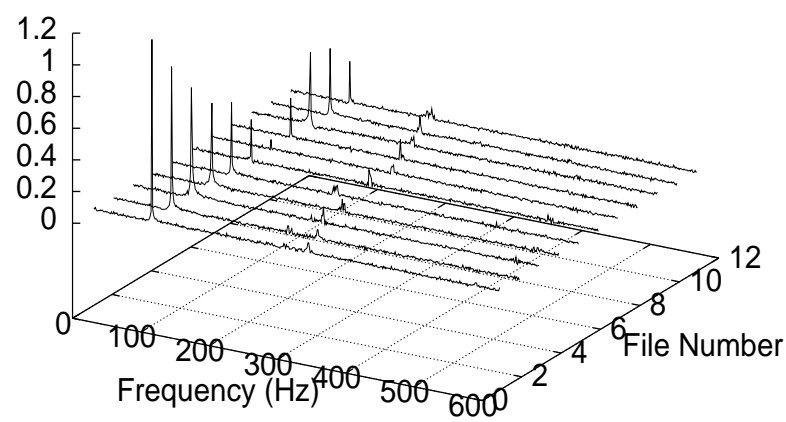

FFT Spectrum of Bunch 5

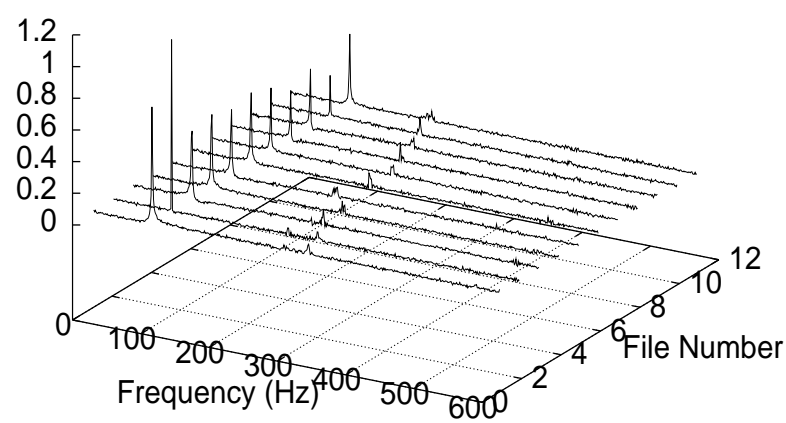

FFT Spectrum of Bunch 7

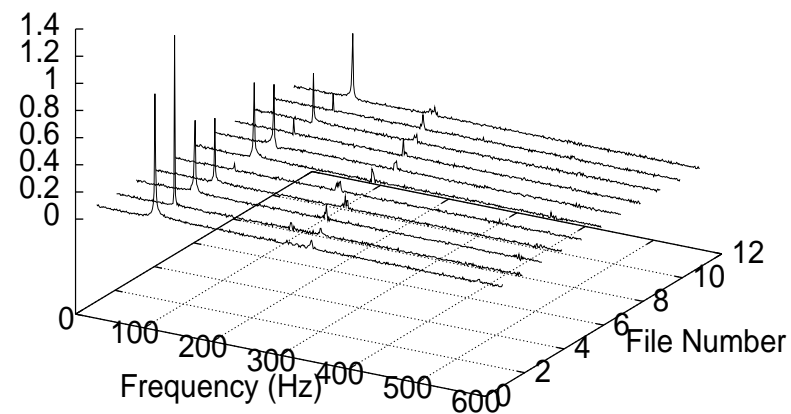

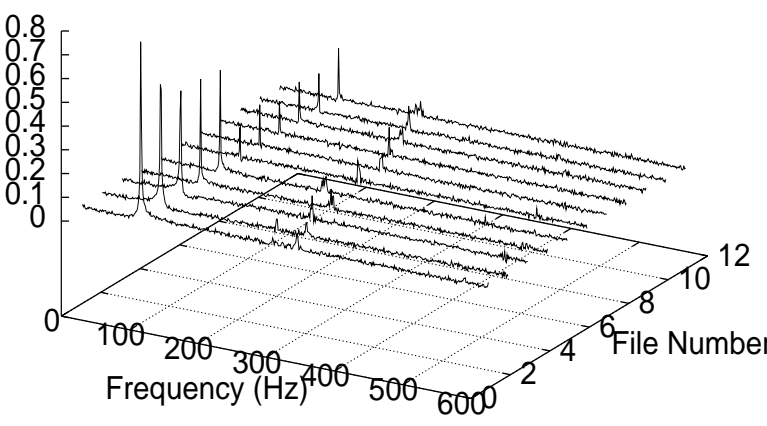

FFT Spectrum of Bunch 4

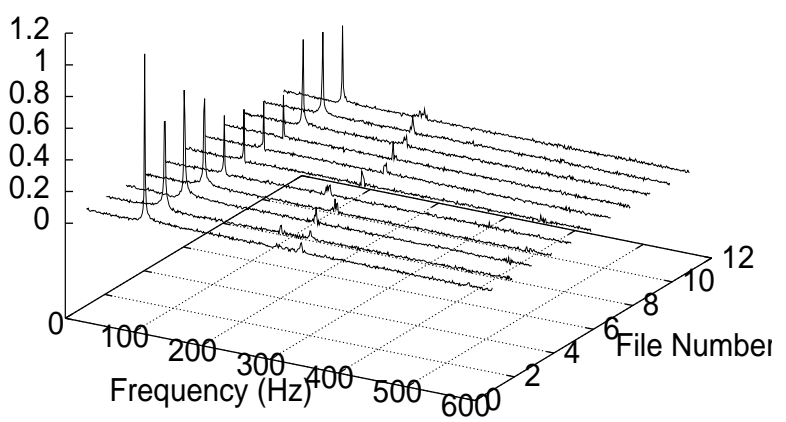

FFT Spectrum of Bunch 6

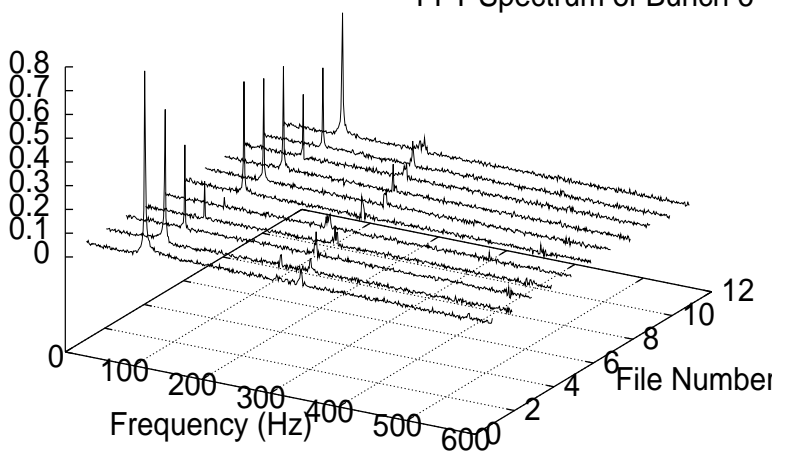

FFT Spectrum of Bunch 8

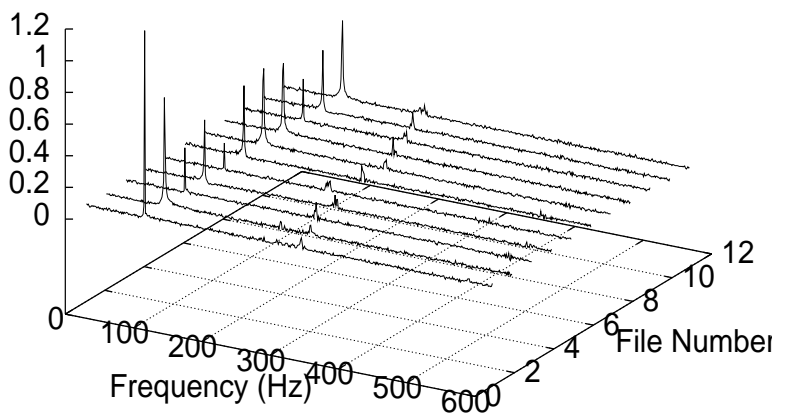

Figure 4: to be continued. 
FFT Spectrum of Bunch 9

FFT Spectrum of Bunch 10
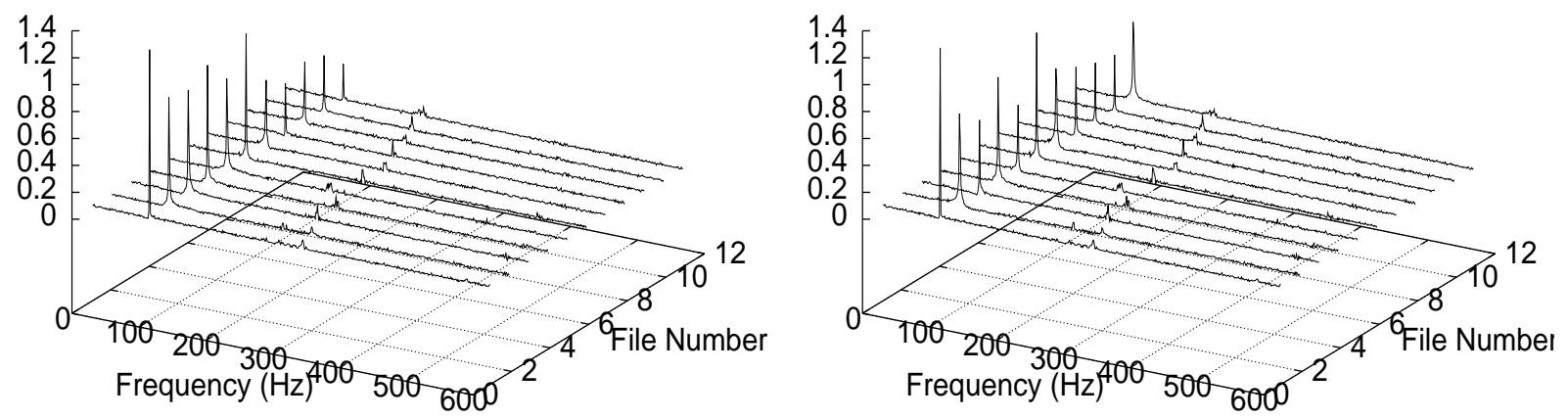

FFT Spectrum of Bunch 11
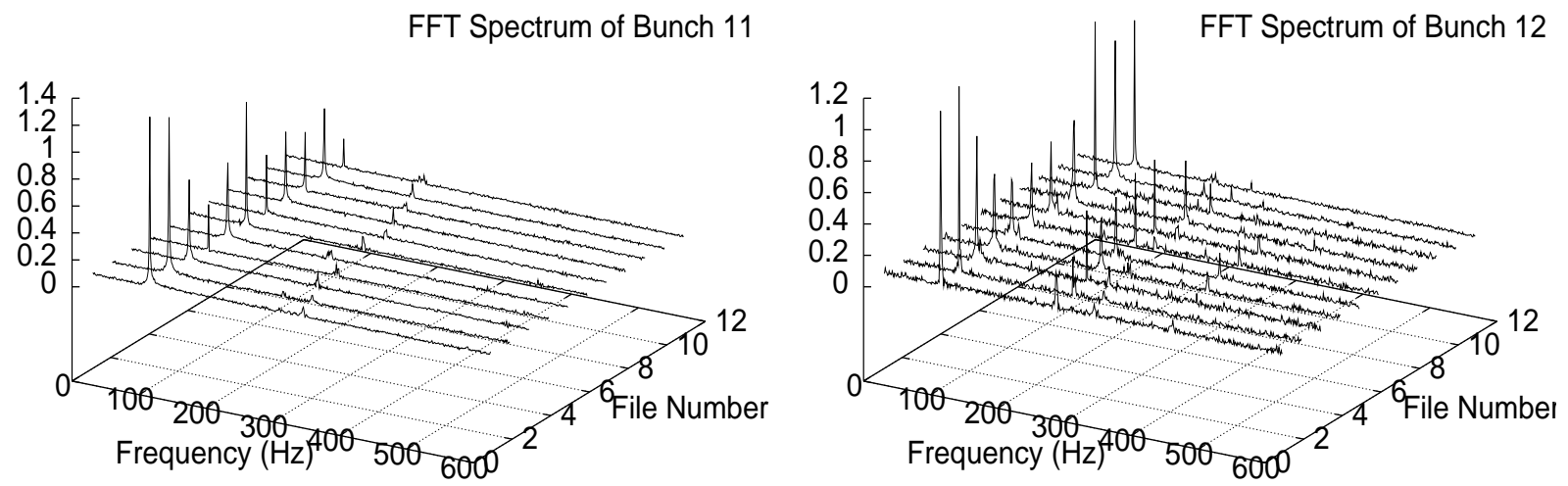

FFT Spectrum of Bunch 13

FFT Spectrum of Bunch 14
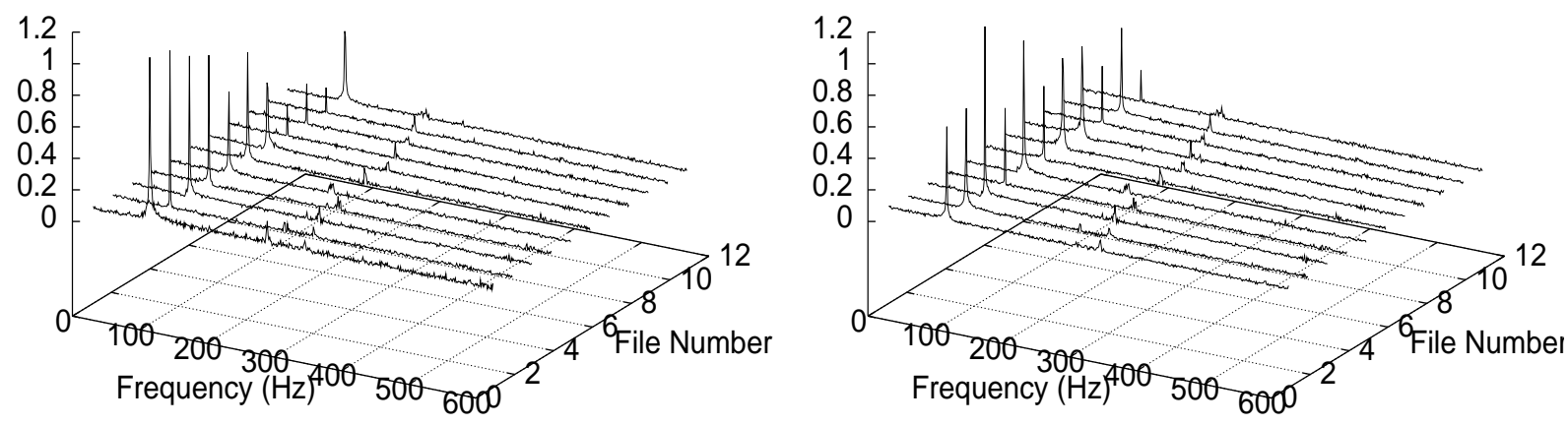

Figure 4 continued: Each plot shows that FFT of one of the 14 bunches in the 11 data files of the second experiment. These 11 data files are each of duration 40960 Tevatron revolutions or $\sim 0.86 \mathrm{~s}$, recorded $0,93,184,275,369,459,549,639,730,821$, and $911 \mathrm{~s}$ after injection. We see a narrow dipole synchrotron peak around $88 \mathrm{~Hz}$ and a broader higher-frequency peak at two to three synchrotron frequencies. Strong third harmonics of the synchrotron oscillation are seen in bunch 12 . 
of the synchrotron oscillation amplitude with time. Since both experiments were performed with both the radial and phase loops open, a possibility of such variations might come from variations of the rf wave. Such strong variation of the synchrotron oscillation amplitude with time is also observed in the FFT spectra of the first experiment Although higher harmonics of the synchrotron oscillation are not observed for most bunches, however, bunch 12 exhibit very strong third harmonic in the second experiment.

FFT was also performed on $\sigma_{t}$ and the spectrum shows only very weak dipole mode or quadrupole mode oscillation. Therefore the higher-frequency oscillation should not be quadrupole or sextupole mode oscillation. Even though there are dipole mode and quadrupole mode oscillations, the beam shows little filamentation in phase space. Consequently, the longitudinal emittance is proportional to $\sigma_{t}^{2}$. Figure 5 shows the bunch lengths of two bunches in the first experiment as functions of time. Although the bunch lengths appear to grow in the first $200 \mathrm{~s}$ and level off afterwards, they are approximately constant for about 1,000 s within the range of the error bars, giving the implication that the emittance is nearly preserved.

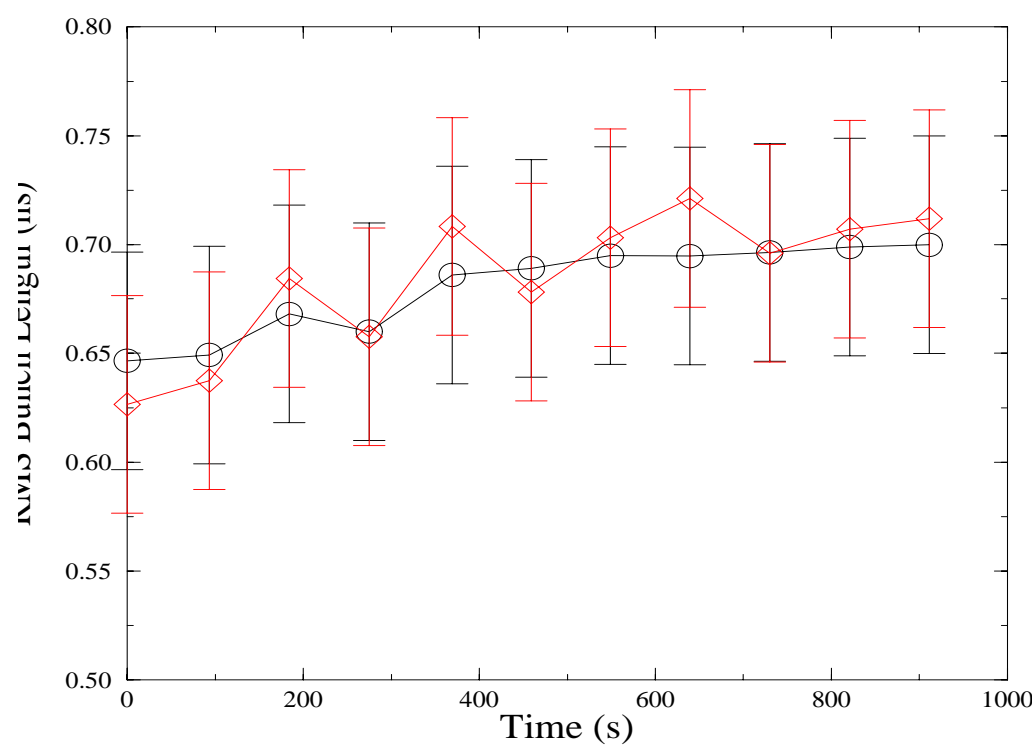

Figure 5: (color) Rms bunch length as functions of time for two bunches in the first experiment: $\circ$ : second bunch, $\diamond$ : 14 th bunch. The longitudinal emittance appears nearly preserved within the measurement error. 


\section{The Dipole Oscillation}

In the second experiment, each data file contains $N=1024$ points separated by $n_{t}=40$ Tevatron revolution. The resolution of the FFT plots is therefore $\Delta f=f_{0} /\left(n_{t} N\right)=1.16 \mathrm{~Hz}$, where $f_{0}=47713 \mathrm{~Hz}$ is the Tevatron revolution frequency, and the spectrum goes up to $\frac{1}{2} N \Delta f=596 \mathrm{~Hz}(239 \mathrm{~Hz}$ in the first experiment where $\Delta f=0.466 \mathrm{~Hz})$. Except for bunch 12, we find all the dipole oscillations fall between 87.9 and $88.5 \mathrm{~Hz}$.

Besides the dipole frequency, we also looked into phase and amplitude of the dipole oscillation. The data points $Y_{n}, n=0, \cdots, N-1$, for the displacement of a bunch center are expanded as

$$
Y_{n}=\sum_{m=0}^{N-1} e^{j 2 \pi m n / N} A_{m}=\sum_{m=0}^{N-1} e^{j\left(2 \pi m n / N+\phi_{m}\right)}\left|A_{m}\right|
$$

where $\left|A_{m}\right|$ and $\phi_{m}$ are the amplitude and phase we are looking for, when the $m$-th discrete FFT spectral line corresponding to frequency $m \Delta f$ coincides with the dipole oscillation peak. They are given by

$$
\left|A_{m}\right| e^{j \phi_{m}}=\frac{1}{N} \sum_{n=0}^{N-1} e^{-j 2 \pi m n / N} Y_{n} .
$$

Since the $Y_{n}$ 's are real, we compute

$$
\begin{aligned}
& b_{m}=\left|A_{m}\right| \cos \phi_{m}=\sum_{n=0}^{N-1} Y_{n} \cos (2 \pi m n / N), \\
& a_{m}=\left|A_{m}\right| \sin \phi_{m}=-\sum_{n=0}^{N-1} Y_{n} \sin (2 \pi m n / N) .
\end{aligned}
$$

The phase and amplitude are then determined by

$$
\begin{aligned}
\phi_{m} & =\tan ^{-1} \frac{a_{m}}{b_{m}}, \\
\left|A_{m}\right| & =\sqrt{a_{m}^{2}+b_{m}^{2}} .
\end{aligned}
$$

Figure 6 shows the phases of the dipole oscillation for different bunches. One can see that there is no phase correlation between bunches. Figure 7 shows the amplitudes of the dipole oscillation for first four bunches. The amplitudes of the bunches show no correlation either. However, the oscillation amplitudes are damped slowly with time with an e-folding time of about 15 minutes. It is worth mentioning that the data points shown in Fig. 7 do not necessarily represent the peak amplitudes of the dipole oscillation. As a result of the finite resolution, the peak of the oscillation frequency usually falls between two discrete FFT spectral lines. The phase, however, is not affected much by the finite resolution, because it is rather insensitive to choosing an adjacent spectral line above below the oscillation peak. 


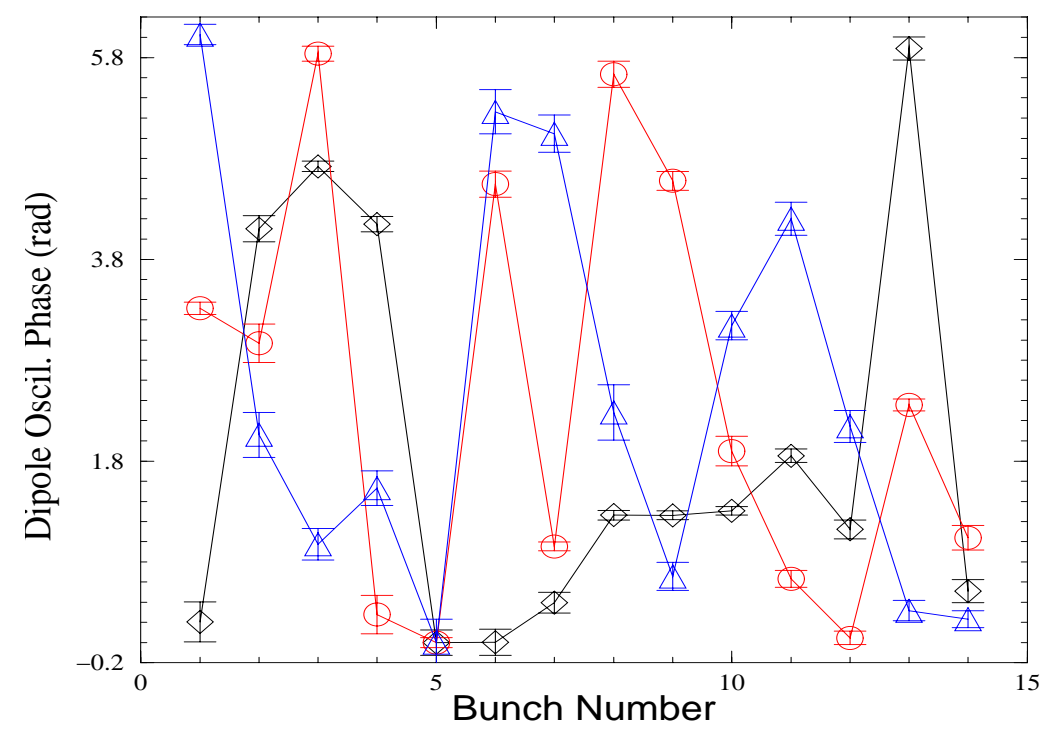

Figure 6: (color) The phases of the dipole mode oscillation of the 14 bunches in the second experiment: $\circ$ : file $821, \diamond$ : file093, $\triangle$ : file184. It appears that the phases of the bunches are not correlated.

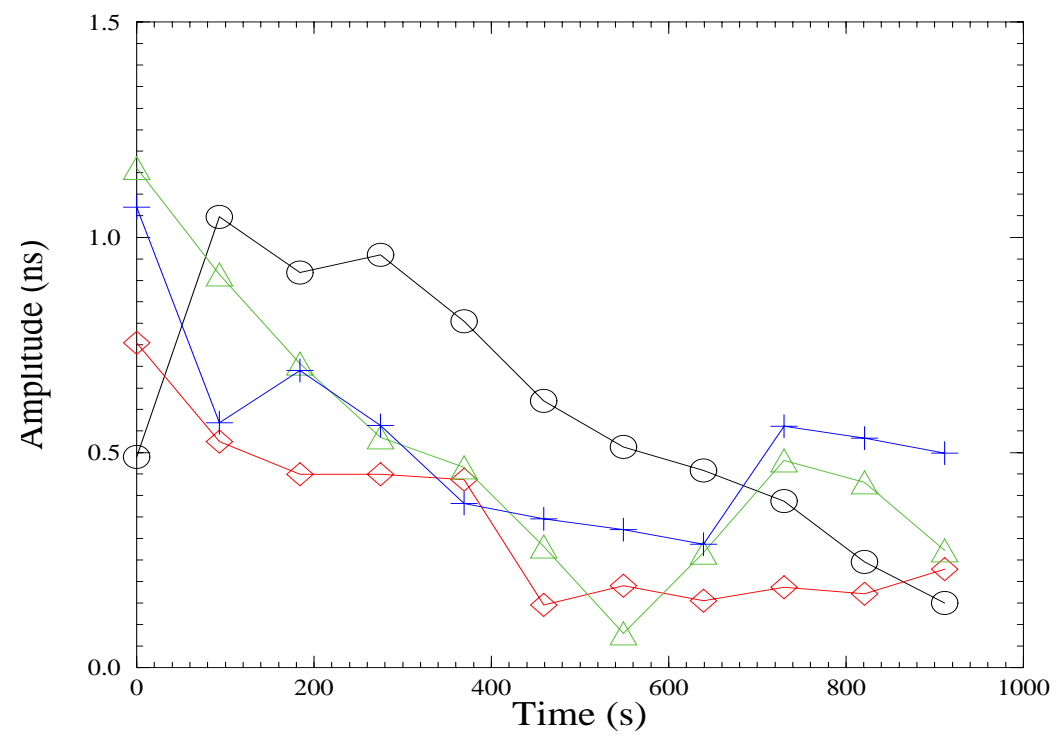

Figure 7: (color) The amplitudes of the dipole mode oscillation of different bunches in the second experiment: $\circ$ : first bunch, $\diamond$ : second bunch, $\triangle$ : third bunch, + : fourth bunch. Although slow, considerable damping is observed. 


\section{The Higher-Frequency Oscillation}

The higher-frequency oscillation is quite different from the dipole oscillation. Figure 8 shows that the higher oscillation frequencies of all the 28 bunches of the first experiment superimposed on top of each other as functions of time. It is obvious that these higher oscillation frequencies are the same for all the bunches, although they become smaller as time progresses. Since the dipole oscillation frequency does not change with time, this provides another indi-

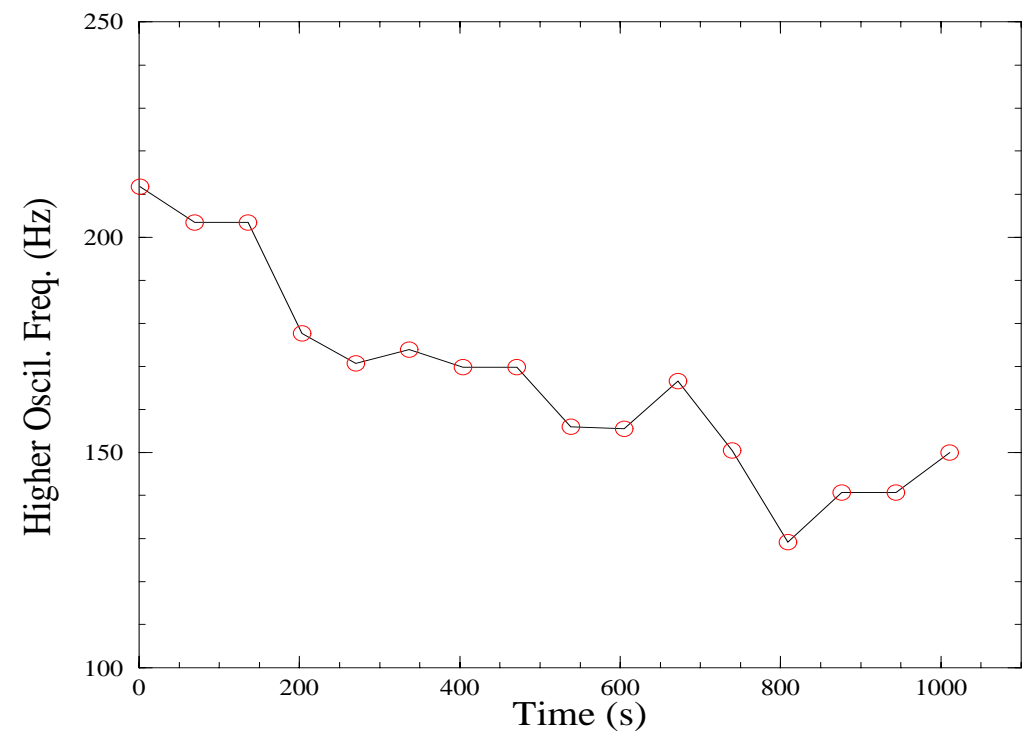

Figure 8: The frequencies of the higher mode oscillation for the 28 bunches in the first experiment. They are all the same and decrease as time progresses.

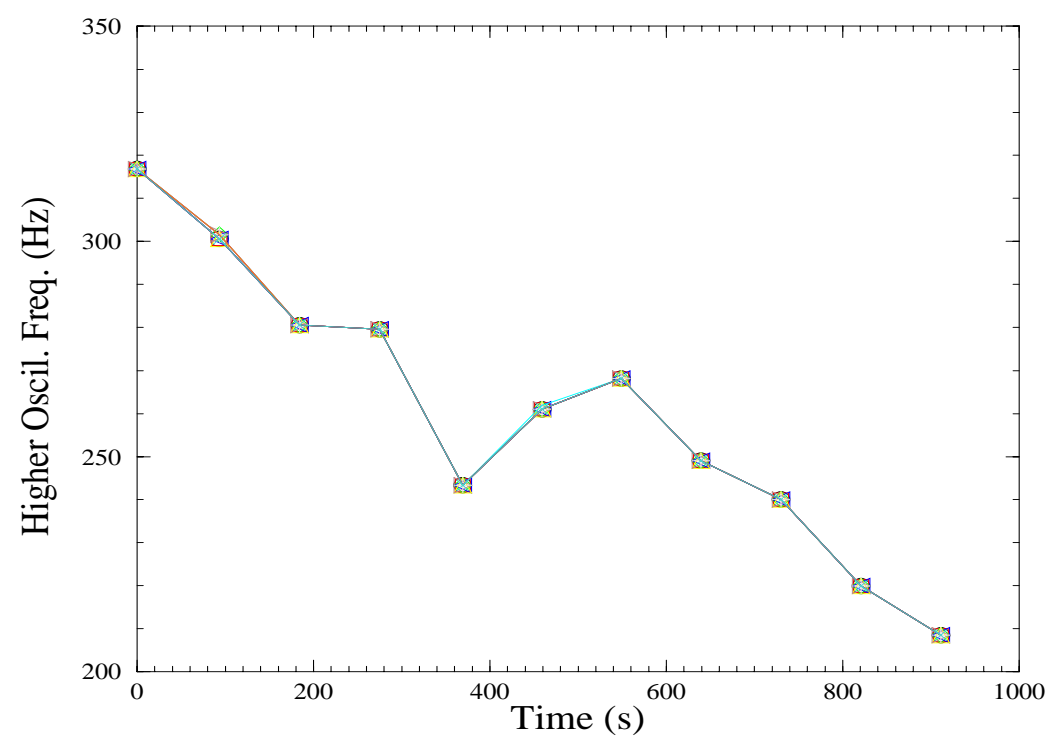

Figure 9: The frequencies of the higher mode oscillation for the 14 bunches in the second experiment. Again, considerable decrease in frequency is evident as time progresses. 
cation that the higher-frequency oscillation cannot be higher harmonics of the synchrotron oscillation. Figure 9 shows the same for the 14 bunches in the second experiment. Again these higher frequencies are the same for all bunches at a certain moment and become smaller as time progresses. However, there is a sharp difference between the frequencies of this oscillation in the two experiments, and those in the second experiment are much larger.

Unlike the dipole motion, the oscillating motion of the higher frequency appears to be coupled for all the bunches. The amplitudes and phases of the bunches were computed according to the routine described in the previous section. The amplitudes of the higher-frequency oscillation of all the bunches are plotted as functions of time in Fig. 10 for the first experiment and in Fig. 11 for the second experiment. Except for bunch 12 in the second experiment, we see that the amplitudes in each experiment are the same for all the bunches at each instant, although they fluctuate in time. Unlike the dipole oscillation, no damping has been observed. The rather large deviations of bunch 12 of the second experiment imply that this bunch oscillates at this higher-frequency mode with much larger amplitudes. The amplitude of bunch 12 at $639 \mathrm{~s}$ after injection has been omitted in Fig. 11. This amplitude cannot be computed with confidence because, as indicated in Fig. 4, the higher oscillation frequency happens to coincide with the third harmonic of the synchrotron oscillation.

Finally, the phases of the all the 28 bunches in the higher frequency mode of the first experiment were studied and the oscillations are found to be in phase at all time. For the second experiment, all 14 bunches were found to be in phase in the higher frequency mode, except for bunch 12 in two data files: data_000.txt and data_639.txt. The phases of the 14 bunches in files data_000.txt, data_184.txt, and data_275.txt are plotted in Fig. 12. For bunch 12 , the higher frequency mode and the third harmonic synchrotron frequency happened to coincide, and, as a result, the computed phase would not be correct. As for the phase deviation of bunch 12 in data_000.txt, no reasonable explanation has been found. Thus, the measurements show that all the bunches (with the exception of possibly bunch 12 in two occasions) have the same features for the higher-frequency oscillation: same frequency, same amplitude and same phase at a given moment. Unlike the dipole oscillation, however, Fig. 11 shows that the frequency of this higher-frequency oscillation decreases with time. In addition, the frequency of oscillation differs a lot between the two experiments. 


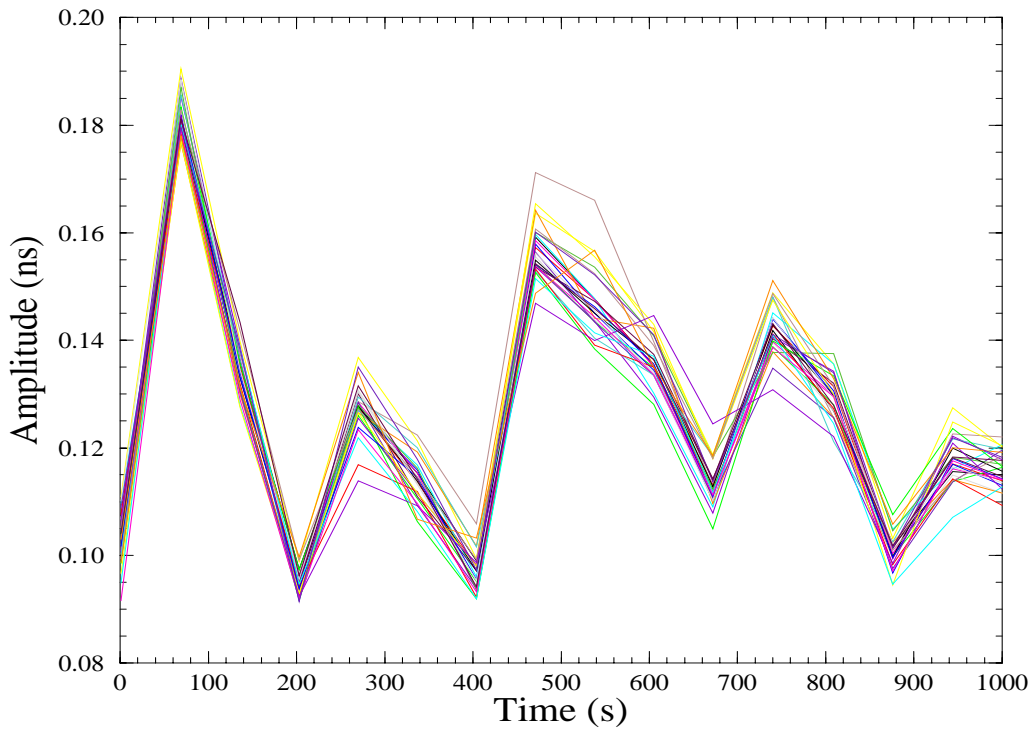

Figure 10: (color) The amplitudes of the higher-frequency oscillation of 28 bunches in the first experiment. Although the amplitude changes with time, all bunches oscillate with the same amplitude at any moment.

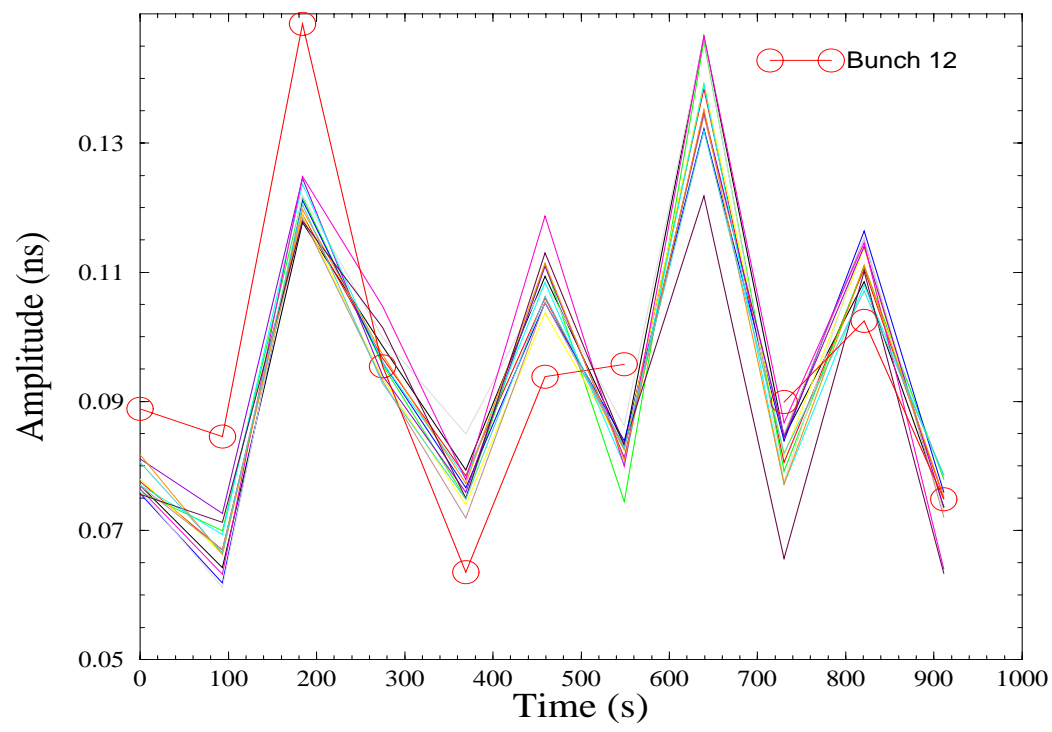

Figure 11: (color) The amplitudes of the higher-frequency oscillation of 14 bunches in the second experiment. Again, the amplitudes of all bunches are the same at any moment in time. 


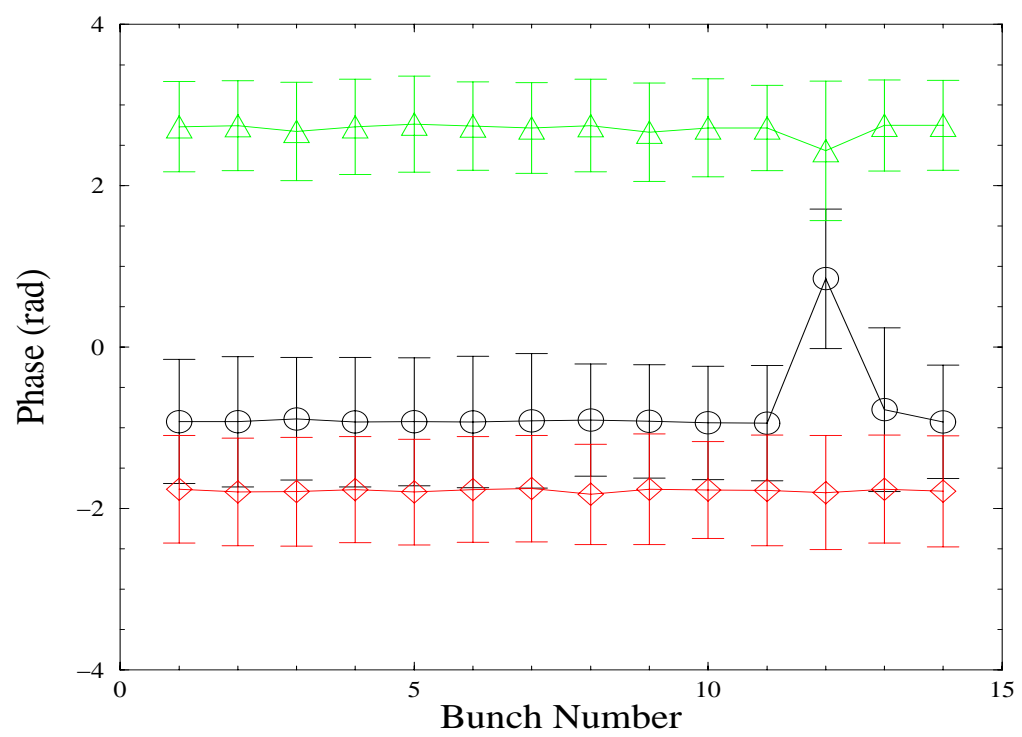

Figure 12: (color) The phases of the higher mode oscillation for the 14 bunches in the second experiment. ○: file data_000.txt, $\diamond$ : file data_184.txt, $\triangle$ : file data_275.txt. Besides the 12th and 13th bunches, the phases of all bunches are the same.

\section{The Unusual Bunches}

In the second experiment, bunch 12 exhibits unusual oscillatory features. The deviations in amplitude and phase from the other bunches have already been shown in Figs. 11 and 12. There are also other usual features, like the exhibition of strong third harmonic, in its spectra as depicted in Fig. 4. In Fig. 13, the spectrum of bunch 12 in file data_184.txt is shown in black, with the spectrum of bunch 10 superimposed in red offset by 0.2 ns. Here, we see a very strong third harmonic together with a weak second harmonic in the 12 th bunch. We also see that even the dipole synchrotron tune of this 12th bunch is somewhat smaller than that of the other bunches. We discover in Fig. 1 that bunch 12 has a much larger oscillation amplitude than the other bunches. That may explain why higher harmonics are observed and the synchrotron frequency is reduced. However, we do not have any explanation of the large oscillation amplitude, except for the possibility of an extraordinary offset at injection.

Besides bunch 12, bunch 13 also exhibits significant third harmonic in file data_000.txt. In Fig. 14, the spectrum of the bunch 12 in file data_000.txt is shown in black, with the spectrum of the 10th bunch superimposed in red offset by $0.2 \mathrm{~ns}$. We see that this third synchrotron harmonic is weaker than that of bunch 12. It is peculiar to see this third harmonic for bunch 13 appear in file data_000.txt only but not in the other files. Besides these two bunches, the third synchrotron harmonic has not been observed significantly in other bunches. 


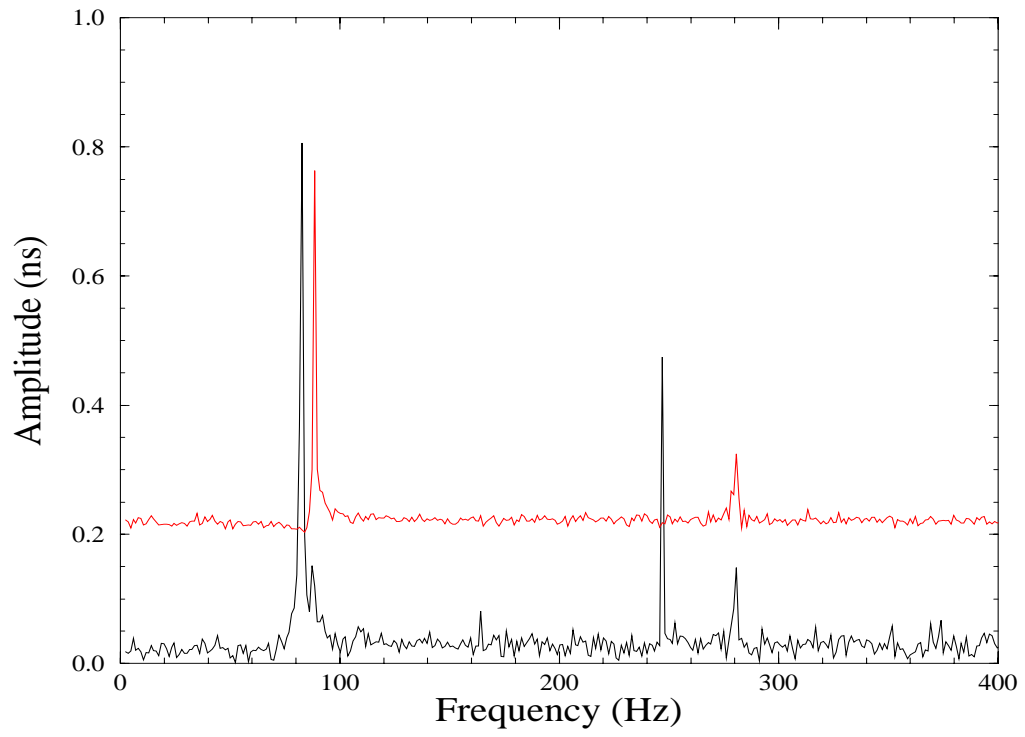

Figure 13: (color) The spectrum of bunch 12 in File Data_184.txt is shown in black. That of the normal bunch (10th) is plotted in red and offset by $0.2 \mathrm{~ns}$ for comparison. The frequencies in $\mathrm{Hz}$ and the amplitudes in ns of these taller lines are: $82.71(0.8061), 247.0(0.4749), 280.7(0.1485)$ for the 12th bunch, and 88.53(0.5636), 280.7(0.1249) for the 10th bunch.

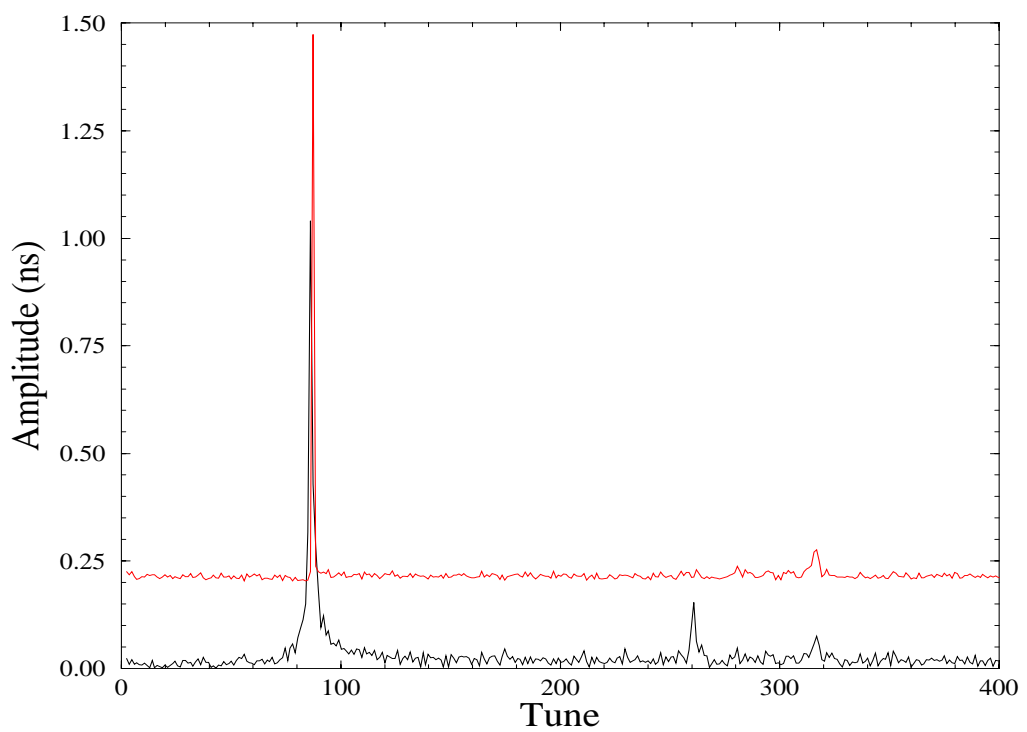

Figure 14: (color) The spectrum of the 13th bunch in File data_0.txt is shown in black. That of the normal bunch (10th) is plotted in red and offset by $0.2 \mathrm{~ns}$ for comparison. Bunch 13 has the third harmonic of the synchrotron tune only in this file. The frequencies in $\mathrm{Hz}$ and the amplitudes in ns of these taller lines are: 86.20(1.041), 260.9(0.1538), 316.9(0.07564) for the 13th bunch, and $87.37(1.274), 316.9(0.0765)$ for the 10th bunch. 
The rms bunch lengths of the bunches were also studied. We plot in Fig. 15 the mean rms lengths of the bunches in each of the 11 data files of the second experiment. We see that the rms bunch lengths of bunches 12 and 13 are much larger than those of the other bunches. Bunches 7, 8, and 9 have lengths similar to those of bunches 1 to 10 and are omitted to avoid making the figure too busy. Special attention should be paid to bunch 12, which continues to lengthen from $\sigma_{t} \sim 0.82 \mathrm{~ns}$ just after injection to $\sim 0.96 \mathrm{~ns}$ around $400 \mathrm{~s}$ before its rapid drop after $650 \mathrm{~s}$. The reason of such change in bunch length is unclear. For the first experiment, no such unusual bunches have been observed.

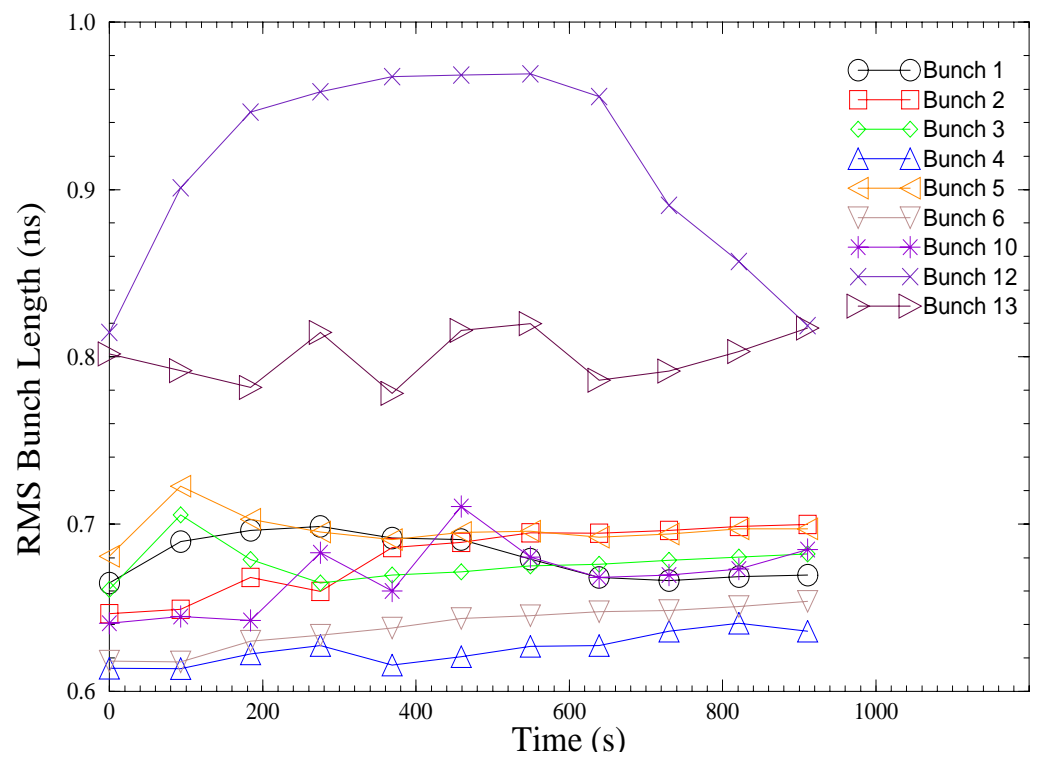

Figure 15: (color) Plots of the mean rms bunch lengths of bunches in the second experiment as functions of time. Bunches 7 to 9 are similar to bunches 1 to 7 and are therefore omitted. Note that bunches 12 and 13 have rms bunch lengths much longer than the other bunches. Also bunch 12 continues to lengthen after injection and becomes shortened eventually.

\section{Conclusion}

We have studied the measurements of the dancing bunches in the two experiments performed on September 21, 2002 and January 12, 2003. Although we have not addressed the cause of the sustained oscillatory motion of the bunches without visible emittance deterioration, some peculiar observations of the dancing bunches have been presented.

With the exception of bunch 12 in the second experiment, the FFT of each bunch shows a narrow peak at the synchrotron tune and a wider peak at a higher frequency, signaling two 
types of oscillatory motions. For the low-frequency oscillation, the frequencies are the same for all bunches and stay the same during the measurement. The phases of oscillation are random among the bunches. The oscillation amplitudes are also different among the bunches although they are found to decay slowly with time. This motion resembles the motion in an oscillatory potential such as the rf potential. In fact, the oscillation frequency agrees very well with the synchrotron frequency of the experiment.

The higher-frequency oscillation has been quite different. Although the frequency is the same for all the bunches, the frequency does drift lower and lower with time. The amplitude of oscillation is the same for all bunches, although it fluctuates with time. All the bunches are found in phase with the exception of bunch 12 in file data_000.txt of the second experiment. This oscillation resembles a forced oscillation driven by some source with a driving frequency falling with time: drifting from a frequency of $\sim 210 \mathrm{~Hz}$ by $\sim 60 \mathrm{~Hz}$ in the first experiment and from $\sim 320 \mathrm{~Hz}$ by $\sim 110 \mathrm{~Hz}$ in the second experiment. We have not been able to identify what this driving source is. Some possible forced coherent oscillations are (1) dipole field time-dependent variation at a dispersive location, (2) quadrupole vibration at a dispersive location, (3) low-frequency rf phase oscillation, etc. So far, the only frequency drifts observed in the Tevatron have been the betatron tunes. Whether this driving source has any relation to the betatron tunes or the betatron tune split requires further investigation. 